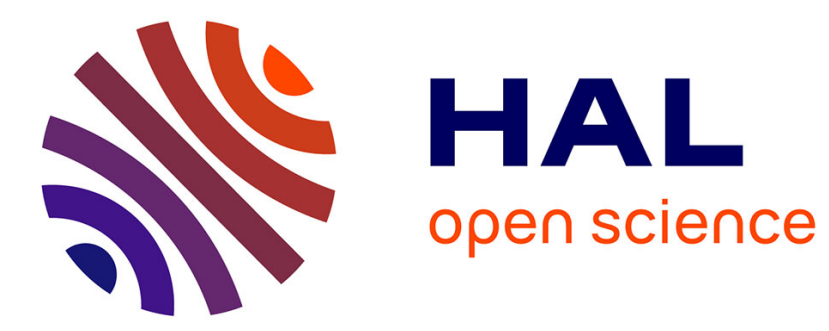

\title{
Authenticity in learning game: how it is designed and perceived
}

Celso Gonçalves, Marie-Caroline Croset, Muriel Ney, Nicolas Balacheff, Jean-Luc Bosson

\section{To cite this version:}

Celso Gonçalves, Marie-Caroline Croset, Muriel Ney, Nicolas Balacheff, Jean-Luc Bosson. Authenticity in learning game: how it is designed and perceived. EC-TEL, 2010, Barcelona, Spain. pp.10. hal00593062

\section{HAL Id: hal-00593062 \\ https://hal.science/hal-00593062}

Submitted on 13 May 2011

HAL is a multi-disciplinary open access archive for the deposit and dissemination of scientific research documents, whether they are published or not. The documents may come from teaching and research institutions in France or abroad, or from public or private research centers.
L'archive ouverte pluridisciplinaire HAL, est destinée au dépôt et à la diffusion de documents scientifiques de niveau recherche, publiés ou non, émanant des établissements d'enseignement et de recherche français ou étrangers, des laboratoires publics ou privés. 


\title{
Authenticity in learning game: how it is designed and perceived
}

\author{
Celso Gonçalves ${ }^{1}$, Marie-Caroline Croset $^{2}$, Muriel Ney ${ }^{1}$, Nicolas Balacheff ${ }^{1}$, Jean- \\ Luc Bosson ${ }^{2}$ \\ ${ }^{1}$ Laboratory of Informatics of Grenoble (LIG), CNRS and University Joseph Fourier \\ 961 rue de la Houille Blanche, 38402 Grenoble Cedex, France \\ ${ }^{2}$ Techniques for biomedical engineering and complexity management (TIMC), University \\ Joseph Fourier, Domaine de la Merci, 38710 La Tronche, France \\ \{Celso.Goncalves, Marie-Caroline.Croset, Muriel.Ney, Nicolas.balacheff, Jean-Luc.Bosson \\ \}@imag.fr
}

\begin{abstract}
A key concern in game-based learning is the level of authenticity games require in order to match what learners can expect in the real world, what keeps them engaged in the game, and what they need to learn. We examined authenticity or credibility in a game from the learner's perspective. There are very few studies on this aspect. We propose that authenticity is the result of a compromise between external authenticity (perceived likeness with real life reference), internal authenticity (perceived internal coherence of proposed situations) and didactical authenticity (perceived relevance about learning goals). Our empirical exploratory study investigated undergraduate students' perceptions of authenticity in healthcare game LoE. First, we suggest some attributes for learning games expected to favour game authenticity. We present the choices made for LoE as result of the compromise between the three aspects of authenticity. Second, we analyze students' behaviour and judgments on authenticity.
\end{abstract}

Keywords: authenticity, credibility, immersion, simulation, role-play, higher education, communication.

\section{Introduction}

Learning games are playing an important role for training people in real world situations. However, an entirely realistic simulation is neither practical nor desirable. On the one hand, game designers introduce realism in a situation by adding more and more elements, and thus bringing complexity to it. On the other hand, when students spend too much time to get familiar with too many details they can skip the main learning goals. In a learning context, a key concern is thus the level of authenticity the game requires in order to have an accurate match of what learners can expect in the real world with what they need to learn. Therefore, authenticity does not mean a perfect reproduction of reality. What need to be authentic are the main characteristics of the type of situations at stake, that is, those characteristics that require learners to mobilize the knowledge targeted in order to be successful in the game. High-fidelity 
simulators do not always lead to better performance in learning therefore when designing a learning situation one must consider an analysis on human perception and information process (e.g. [1]). Authenticity is both a function of the game and the perceiver, and we shall define them in the following, starting from a brief overview of related concepts.

Research in education started problematizing authenticity about a century ago, including the work of John Dewey who studied the relationship between learning and experience. To define authenticity in the context of learning games, we consider recent research on new technologies (see also [2]): video game research on immersion and engagement of players, human-computer interaction research on credibility of various computer environments, and communication research on perception of television content.

First, in video game research, many terms have been developed to try to account for these experiences, such as perceived realism [3], and immersion ([4], [5]). One mean to create authenticity in learning games is immersion, making learners feel a certain situation to be real although they know it is not. According to Brown [4] the main indicator of immersion by the user's perspective is the player degree of involvement. Game designers know players may engage in different ways in a game, mobilizing themselves differently, whether they are challenged on their ability to act rapidity, to find the best strategy, to communicate with different people, etc. To immerse in a game is to get involved in the context, not only physically but also mentally and emotionally. There have been numerous definitions of immersion in games based on different aspects of involvement (e.g. tactical, strategy or sensory immersion [5]). In this paper, we focus on immersion in a simulation of an authentic situation, and more specifically, a situation that involves many moments of interactions with people. We call "interactional immersion" such an immersion that relies mostly on interactions with other players or characters of the game [6]. We use engagement as one of many indicators of perceived authenticity, i.e. whether students play the game or not.

Secondly, authenticity can be linked to the concept of trust as developed in Human Computer Interaction studies, the second field of research from which we attempt to define authenticity. The use of the word trust refers to credibility as in "trust the information" or "believe the output", a meaning that is relevant to authenticity [2]. Tseng and Fogg [7] suggest various terms to assess trust or credibility in computer environments: believable, truthful, unbiased, reputable, well-intentioned. According to these authors there is a direct connection between "perceived lack of veridicality" (which we relate to authenticity) and lack of credibility. In the present paper, we shall treat perceived authenticity and credibility similarly. Indeed, users do not always have a personal experience of the simulated reality. The question of authenticity is thus: do they believe it or not. We will discuss in particular the credibility of the feedback from the environment to the learners.

Thirdly, in communication and media studies, several researchers have examined the perceived reality, or modality judgments, of television content ([8], [9]). These researchers identified various criteria involved in viewer judgments regarding the reality (or the realism) of media content. Chandler [8] proposes four criteria: possibility (possible to do in reality), plausibility (possible to do but highly unlikely that you would do it in real life), actual existence (could be done but nothing like this 
actually exists in reality), and constructedness (this is just a virtual situation and not a real one, it is pre-constructed). Interestingly, the judgments are measured on a likelyhood scale, which relates to the concept of credibility cited above. Furthermore, Brusselles and Bilandzic [10] offer a theoretical framework to explain circumstances under which perceptions of "unrealness" affect engagement in narratives and subsequent perceived realism judgments. They discuss three types of unrealness: fictionality, external realism (match with external reality), and narrative realism (coherence within a story). They show evidences that fictionality does not affect narrative processing unlike the two others.

We now come to a definition of authenticity that will be used both to design game authenticity and to analyze learners' perceived authenticity. We keep the notions of external and internal authenticity [10] and add a new one specific to our context of learning game, that is, didactical authenticity [6]. As summarized in figure 1, a learning game may be designed, and later perceived, as authentic from three points of view: it can be more or less realistic (perceived likeness with a real life reference), coherent (perceived internal coherence of the proposed situations) and relevant (perceived relevance with respect to learning goals).

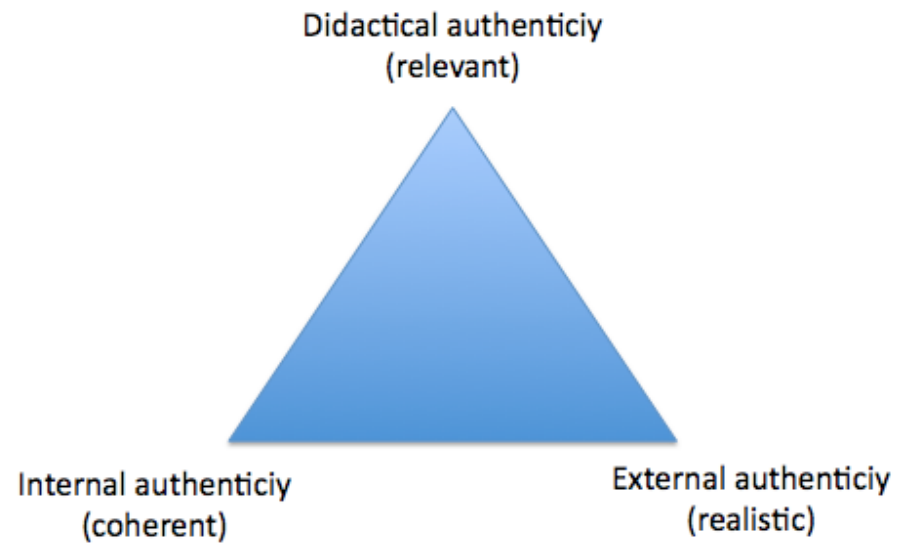

Figure 1. Model of authenticity

Why should a learning game be authentic? Some fields are difficult to teach because students do not immediately see the purpose; they are not motivated because they do not relate the learning goals to their personal training project. This is often the case with mathematical teaching contents (e.g. statistics for medical students like in the game described in this paper, calculus for experimental sciences, etc). Like other approaches do (e.g. inquiry learning, problem-based learning), game-based learning gives an authentic context to the learning at stake. In our case, namely learning statistics, students will face a public health issue rather than directly a statistical issue. We shall now justify each of the three dimensions of the model (figure 1) one by one.

First, the context above mentioned should be authentic in the sense of external authenticity at least for professional training. Otherwise, students would not have the feeling of being prepared to react adequately in real professional situations. Second, 
the game must remain consistent with a logical sequence of events and feedback. This refers to the internal authenticity. Inconsistencies in the game may cause disengagement. Moreover, communication and media research suggest that one is more susceptible to persuasion or abstract lessons, when engage into a narrative. Third, didactical authenticity is related to appropriation, the fact that learners make the problem proposed their own, looking for their own solutions rather than finding strategies to do only what the teacher expects [11]. This will allow the learner to appropriate the meaning of the situation, i.e. what is the point in learning terms. Otherwise, the learner is just focusing on a particular solution of a particular problem with little ability to transfer to other similar problems.

After this definition of the nature and scope of authenticity, we introduce below the case study on the game LOE exploring various judgments expressed by learners after the game as well as their behaviour during the game. The following research questions guided our investigation:

(1) Do students perceive the game as authentic? How do students behave in (authentic) moments of interaction with characters of the game?

(2) What cues (within the game environment) enable students to make judgments of authenticity? What roles do these cues play in students' judgments?

\section{Method}

\subsection{Description of the game environment used in the study}

The Laboratorium of Epidemiology ( (LOE) immerses learners into a full-scale, persistent and distributed simulation combined with a game scenario [6], [12]. It was collaboratively designed and used by researchers, teaching staff (hereafter called tutors), and students as both an educational project and a research project. The educational project forms part of a medical school course in biostatistics. The research project, to which we gave the name "Laboratorium", allows repeated data collection campaigns that are not unique events in students' or tutors' lives and is an attempt to reduce data collection biases and produce well-documented databases.

The game is based on a computerized simulation of various institutions (including hospitals) and role-play. Students play the role of public health physicians and are placed in an otherwise inaccessible professional situation involving the occurrence of a disease in several hospitals. Students have to design and carry out an epidemiological study, and write a scientific article to be presented at a congress. The main learning goal concerns the statistical analysis of a medical database. However, students are given a mission that contextualizes the problems of doing statistics. The mission is to design a diagnosis tool for VTED (Venous Thrombo-Embolic Disease) for hospital use. While working on this problem, students will learn statistics, understand the role they play, and more generally the function of statistics in public health.

The game is fully integrated into the standard medical school curriculum. It lasts four months including eight four-hour sessions in class. The main tasks for students, the learning goals and the main part of the computer environment connected to these 
tasks, are indicated in the following table. It is structured by the eight in-class sessions (left-side column). These tasks are performed in teams of three to four students.

Table 1. Analysis of the main tasks and learning goals of the game

\begin{tabular}{|c|c|c|c|}
\hline & Main tasks & Goals & Computer environment \\
\hline 1 & $\begin{array}{l}\text { Bibliographical } \\
\text { research, choose the } \\
\text { main objective and } \\
\text { make a plan }\end{array}$ & $\begin{array}{l}\text { To gain knowledge about a specific } \\
\text { disease as well as the } \\
\text { methodologies used in } \\
\text { epidemiological surveys }\end{array}$ & $\begin{array}{l}\text { Virtual websites of various } \\
\text { organization }\end{array}$ \\
\hline 2 & $\begin{array}{l}\text { Design an } \\
\text { epidemiological survey } \\
\text { and sent the protocol } \\
\text { for validation }\end{array}$ & $\begin{array}{l}\text { To construct and structure a survey } \\
\text { (sample quality, indicator quality, } \\
\text { ethical considerations, etc.) }\end{array}$ & $\begin{array}{l}\text { E-mail application, Ethical } \\
\text { Research committee } \\
\text { website }\end{array}$ \\
\hline 3 & $\begin{array}{l}\text { Carry out the survey at } \\
\text { one or several hospitals }\end{array}$ & $\begin{array}{l}\text { To implement a survey (translate } \\
\text { patient saying into data, control and } \\
\text { organize data) }\end{array}$ & $\begin{array}{l}\text { Virtual hospitals (online), } \\
\text { Mobile phone }\end{array}$ \\
\hline $\begin{array}{l}4 \\
5\end{array}$ & Analyze data & $\begin{array}{l}\text { To analyze data with statistical } \\
\text { tools and thus understand them }\end{array}$ & $\begin{array}{l}\text { Statistical software, } \\
\text { Spreadsheet application }\end{array}$ \\
\hline $\begin{array}{l}5 \\
6\end{array}$ & $\begin{array}{l}\text { Write an article and } \\
\text { submit it to a congress }\end{array}$ & $\begin{array}{l}\text { To understand how a scientific } \\
\text { article is structured, and how to } \\
\text { select and present evidences } \\
\text { (statistics may come in different } \\
\text { forms) }\end{array}$ & $\begin{array}{l}\text { Text editor, Congress } \\
\text { website }\end{array}$ \\
\hline 7 & $\begin{array}{l}\text { Prepare an oral } \\
\text { presentation }\end{array}$ & $\begin{array}{l}\text { To synthesize results taking into } \\
\text { account a reviewer's comments }\end{array}$ & $\begin{array}{l}\text { Slide editor, Congress } \\
\text { website }\end{array}$ \\
\hline 8 & $\begin{array}{l}\text { Attend a (simulated) } \\
\text { medical congress }\end{array}$ & $\begin{array}{l}\text { To defend orally their decision } \\
\text { tools based on statistical arguments }\end{array}$ & \\
\hline
\end{tabular}

One important challenge in this project was to design interactions with characters of the game that help students to appropriate different problems, acquire skills and engage in the game [6]. The system of interactions of Loe was inspired by recent work on embedded phenomena [12] and participatory simulations [13]. It is a persistent and distributed simulations, sharing several characteristics of pervasive games [14].

Between sessions 2 and 3 (table 1), students have to ask for authorization from the Head of each medical department of the hospital they wish to visit, in order to be able to interview patients. For this purpose, they have to make a phone call and, having listened to a brief message, they have to formulate their request in the way they think most appropriate. Later, a tutor listens to their message and replies by SMS giving them either permission or a documented refusal. A team of students can make several 
calls, either to have access to several departments, or to repeat their request after a refusal.

Students use their personal e-mail application on various occasions. For example, between session 2 and 3 (table 1) they send their protocol for validation by the Research Ethics Committee. They have access to the (virtual) website of this committee that gives an email address. This is compulsory since only approved protocols can be implemented at the hospital. The tutors behind these characters (experts of the Committee) use dedicated e-mail addresses and Webmail applications to answer to students.

During session 3, students interview patients in simulated hospitals each containing three wards and five patients per ward. The system allows the students to interact with the simulated patient by selecting one of five preformed questions: present illness, present lifestyle, past history, family/social history, and medications. For each question, an appropriate video clip provides the interviewer with the patient's reply. These clips are based on scripts made from real patient files: National Survey in Hospitals in France managed by J-L. Bosson.

In order to be able to participate to the simulated medical congress (session 8 in table 1), students have to upload their article on the virtual congress web site (between sessions 6 and 7). This is the least represented character of the game since not even a name is given to him/her. Later they will get a report on their article, visible on the web site. In many cases, they will be asked to revise their article and send it again. Articles are divided into two groups by the organizers of the congress and best articles are given a longer time of presentation.

The challenge was to make these interactions authentic according to the three dimensions of figure 1 . Interactions should evokes the ones one would meet in a real hospital including feelings and constraints (realistic), they should also be useful and timely within the mission proposed (coherent), and finally they should help students acquire the knowledge and skills at stake (relevant).

\subsection{Game attributes with regards to the authenticity model}

We now give a list of attributes of the environment that were used to make the students' experience more authentic [6]. The game environment components were designed with different compromise within the triangle; part being predominantly on the realism dimension, other part being more oriented towards either the coherence or the relevance dimensions.

Table 2 summarizes nine attributes of authentic learning games based on a mission that implies interacting with characters. Below we give an example in LOE and an analysis using our model (figure 1).

Table 2. Environment attributes of authenticity

\begin{tabular}{l|l}
\hline Themes & Attributes \\
\hline
\end{tabular}




\begin{tabular}{l|l}
\hline Mission & $\begin{array}{l}\text { Mission content and resources } \\
\text { Original data }\end{array}$ \\
\hline Mise en scene & $\begin{array}{l}\text { Graphical representation } \\
\text { Structure of the environment }\end{array}$ \\
\hline User freedom & $\begin{array}{l}\text { Constraints } \\
\text { Level of control of the users }\end{array}$ \\
\hline Interactions & $\begin{array}{l}\text { Characters' personification } \\
\text { Behaviour and feedback from characters } \\
\text { Mode and media of communication }\end{array}$ \\
\hline
\end{tabular}

A mission. When starting the game, students are given a mission that gives context to the problems designed for learning. This mission has been designed to mimic a real situation with the help of an epidemiologist who conducted this mission in the past. It is presented to students in the form of text and video (of a meeting between professionals) on the commission's web site. This commission is the institution that gives the mission. We used real data (patients files) as stated above. Therefore, the external authenticity was the most important to design these attributes related to the mission.

Mise en scene. The environment that provides a mise en scene is for most parts online. Students find the websites of the different organizations they have ot interact with. This is designed following mainly the internal coherence principle. Indeed not all the organization one could encounter in real life are represented but the interactions are designed to flow logically within the game. Furthermore, the graphical representation, especially of the hospital, follows the didactical authenticity dimension. Instead of an avatar moving within a represented hospital with corridors and wards, we kept only those parts that are relevant for learning, in particular the patients talks (scripted).

User freedom. First, the constraints of the interaction may be considered. For instance, in the interaction with patients, it is important that a patient does not repeat at will his/her answers, which is a constraint that was reproduced in our system. This particular constraint is designed for didactical authenticity. Indeed, when a patient does not repeat oneself, students learn that they need to prepare their interview and also to look for ways to control their data, two learning goals of the course. Second, the level of control of the students on environment and tasks had to be defined. In LOE, the tutor is accompanying the students who discover by themselves what they have to do and how they can navigate into the environment. Students are physicians who can ask for help but who are relatively free otherwise (external authenticity).

Interactions with characters. Considering the authenticity of our system of interaction, we identified three attributes (see table 2). First, the characters students are interacting with may be more or less authentic. This is decomposed into two 
issues. First, the authenticity of the character refers to its personification: what information is available and in what form (text, photos etc). This is limited in LOE to satisfy mostly internal authenticity. Second, the content of the feedback the character gives to students may be more or less authentic, depending on how adapted to student's actions this feedback is. For didactical authenticity, this feedback is communicated verbally or otherwise (e.g. a patient with heavy breathing can be an important sign) and relevant or not (students need to sort information). Finally, another issue is the channel of communication (by email, phone, etc) and the mode (textual, verbal, visual). The latter was designed for external authenticity.

\subsection{Data collection and data analysis methods}

Our sample is composed by 170 second-year students of the medical school of Grenoble, France, in a compulsory biostatistics course they followed in 2009/2010. They are distributed over 45 teams of 3 or 4 students. Our first data set consisted of messages left on the answering machine of the hospitals by the 45 teams. There were 167 voice messages in total. Our second data sets consisted of transcripts of phone interviews with volunteer students. There were between 19 and 23 students from as many teams (one student per team) interviewed after each session.

We shall now explain our method with regards to the research questions asked. This is mostly an exploratory empirical study with two goals, one is to get a large variety of students' feedback, the other is to collect both what they do and what they say. For these reasons, we got data both during the game (students interact with characters of the game without explicit presence of researchers) and after (students talk to researchers and reflect on what they did). To answer question (1) on student perceived authenticity, we first analyzed student' behaviour as they interact with characters of the game. The goal was to look for signs of engagement into their role, in other words, see whether students behave as if the situation was real and/or useful for their training. Secondly, to answer question (2) on cues that enable students to make judgments of authenticity, we analyzed the phone interviews. We asked three questions during a phone interview that occurred about an hour after the end of each session: (i) what did you produce today, (ii) do you perceive what happened today as intuitively credible with regards to a professional reference or strange, and (iii) do you think what you did today useful for your professional training. Very little was said by the researcher-interviewer in order to let students spontaneously put issues on the table. In particular, participants were not notified about the realism of the environment (e.g. that data were real) or about how it would be for a professional.

Method of analysis of the voice messages. In a real life situation, the voice message would be formally addressed to the head of the department, indicating a relation of hierarchy which in turn fits the context proposed in this part of the game. Therefore, we could expect students to introduce themselves under the role of a medical doctor since this is the way they are assigned in the game by the public health commission "you are a doctor...". Here are the criteria used:

Attitude: their attitude towards the head of the hospital department (Formal or Informal). 
Identity: the name and the role under which students introduce themselves (Medical Doctor, Medical Student or Themselves).

Context information: students give contextual information on the mission (e.g. the various organisations) or, conversely, on the course. Specify the objective of their survey to justify the request.

We also collected time and date of the phone call.

In order to analyse the nature of these messages we classified them into four groups: Successful, Unsuccessful or Partial, Mislead and Tryout massages.

Successful messages qualify the ones able to request an authorisation as well as to report students' main objective of their survey or at a pinch to mention it concerns a survey about the thromboembolic disease.

Unsuccessful or Partial messages: Unsuccessful messages were unfinished messages, incomplete messages (when students failed to identify themselves or their group), and ineffective messages (when students, despite requesting authorisation, failed to report a survey main objective). Partial messages were rectification partial messages or new authorisation request partial messages (when in a subsequent message students required an authorisation to access another hospital department without giving details of their survey oftentimes refereeing to a previous message).

Mislead messages were messages that presented only an authorization request following the instruction on the answering machine.

Tryout messages when students hung up the phone immediately after hearing the answering machine message.

A first grid of analysis emerged from previous pilot study. For this year, messages were coded by a $\mathrm{PhD}$ candidate in Cognitive Sciences and a $\mathrm{PhD}$ in Applied mathematics. This collaboration resulted in an adjustment of the criteria to the present sample through trials using 15 messages. Further on, we compared the results of 43 messages in order to check if there were any incongruities. Finally we double checked 167 messages and discussed a few incongruities especially concerning the classifications nature of some messages.

Method of analysis of the phone interviews. The phone interviews were transcribed. Then each unit of sense was associated to one of the nine game attributes (table 2), if any. There were approximately 8 hours of telephone interviews (representing all four first sessions of Table 1). Each unit of meaning (a topic addressed by a student after question) was then allocated to one of the nine attributes, when one of them was mentioned by the student. The analysis goal was to identify cues mentioned by students to explain a perception of authenticity (i.e. if a task seemed credible or not and why). We can also identify the positions taken by a majority of students, as well as individual variability. 


\section{Results}

\subsection{Analysis of the voice messages}

The majority of the students (115 out of 136 messages that were not simple tests) have a formal attitude. This could be a sign of perceived authenticity by the students, whether it is because the task is realistic, coherent or relevant is another issue. However, there were 21 calls showing an informal register (mainly laughter), which would not have been allowed in reality (if calling a real hospital). Also, no team has assume the identity of a doctor, and only 40 of 136 callers introduced themselves as students. The 96 (71\%) remaining, did not play their role as a doctor and did not even give sufficient status to justify their request. However, they all gave their name. Finally, 20 of 136 messages from 13 different teams told about the bio-statistics. None of the messages mentioned the organization they are working for in the game.

We looked for expected messages to assess students' perception of the relevance of the teaching situation, that is, those able to meet the educational goals. These messages should at least include information on the identity of the caller, the name of the department where the student wants to interview patients and the presentation of the main objective set by the team, all in a relatively formal language. If any of these criteria is not validated (e.g. informal attitude or lack of main objective), the message is rejected. The remaining messages indicate that the student perceived the issues underlying teaching (didactical authenticity). Among the 167 messages, 62 (37\%) are coded as expected messages.

Each team has recorded several messages: 3 messages on average up to 17 messages for the same team. This shows their need to better understand the situation and the expectations of the game before reaching the goal. The evolution over time of the overall content of the messages, however, is positive: the five weeks the students have filed messages (see Figure 4), the proportion of messages considered expected grows from $15 \%$ to $50 \%$. The messages of the first week were mostly incomplete $(60 \%)$. However, eleven teams (of 45) leave no message corresponding to our expectation, during the five weeks.

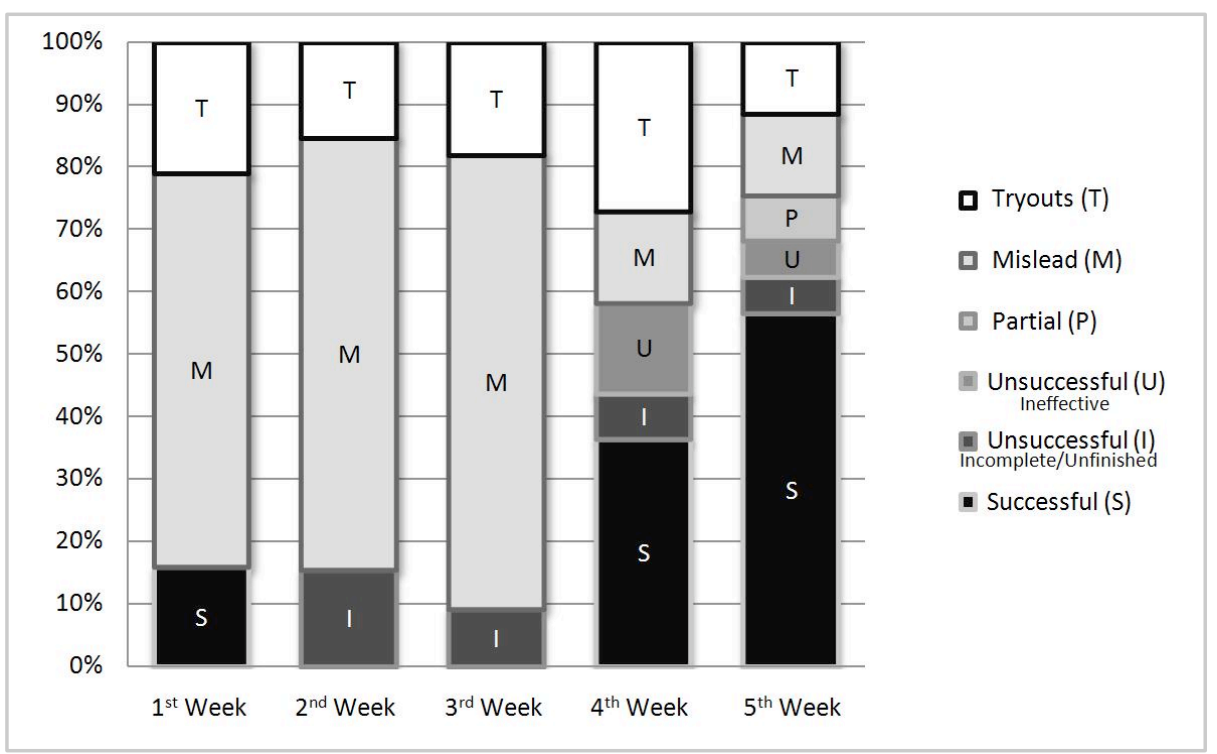


Figure 2. Distribution of the phone messages nature across 5 weeks.

To conclude, our combinations of indicators showed that most students did not perceived the situation as realistic nor relevant. These results will be confirmed by the comments made by students during phone interviews.

\subsection{Analysis of the phone interviews}

The following presentation and discussion of results is organized around the four groups of attributes of table 2. In keeping with the study's exploratory aim, we believe that the worth of our findings rests on the diversity of issues tackled and on a detailed exposition of differences among individual cases. Therefore, qualitative assessment of the relative importance of various issues for different individuals was a prevalent underlying process in our analysis.

A mission: given resources and original data. Students made judgments concerning the game mission mostly during the very first phone interview, at the end of the first session (table 1). Different cues were important to different participants and assessments of the game's authenticity diverged depending on what cues were perceived or taken into account by different individuals. On one hand, some students were ready to engage in the mission and believe in its authenticity. A realism comment from one of the student is "it looks like it was not made just for us". Some students said that the documents themselves look "serious". Students mentioned most often two cues: the figures in these documents, or the model of protocol, both look real. The video showing a meeting between physicians and introducing the issues of the mission was also taken as a cue by several students. One students added that it shows "... the importance of teamwork; it is not just a person who is going to change the world".

On the other hand, some students realize that they are going to make their own study but they know the results in advance (from a literature search in the virtual library). They think that they will "not be able to prove anything new" or that they have to "pretend that the patients are going to be interviewed but know that data have been collected already". The latter points out an internal incoherence perceived by some students: they have to write a protocol as if they were going to collect data, but at the same time they believe (at least for this session) that data will be given to them.

The data that are sent to student in the form of a table with patient in lines are real but students do not know it and most of them think that they are not but "look very real". Different cues are used to suggest the authenticity of the data: "the data are variable, with few repetitions", "there are many information", "it looks like a physician did the survey because all the different forms of the disease are distinguished", "there are a lot of details on each patient", “ we did not get exactly what we wanted, it is not ideal, it is annoying", "the statistical test shows what we expected", "we got unexpected result". The latter shows that whatever the result, students do not question the authenticity of the data. This and the impact of the figures included in the document show the importance of figures as a crucial cues for student of the authenticity of their mission. One student even said that if the result is 
not what is expected from the literature review they will have to look for an error in their methodology.

Mise en scene (visual and graphical attributes). No comment is made about the site structure and graphical design. It may be that these aspects are not a source of evidence of authenticity for students. They appreciated the quality of the environment, but this raised not judgement on its authenticity (external, internal or didactical).

User freedom (constraints and level of control). One of our findings in this area is that a number of participants mentioned the constraints spontaneously and could even discern the value of the constraints with regards to one of the authenticity dimensions. For one student, two cues for what we called external authenticity were the fact they do not "have access to patients without asking for an authorization" and that " the more in advance they ask for data, the larger the number of patient files they get". By contrasts, for those students who got data too quickly, the interaction was not realistic. One important constraint with a didactical authenticity was the fact that students could not listen twice to the same patient answer. None of the students understand the value of this constraint (see previous section) and several of them even thought that it was not realistic since in real life it is possible to ask a patient to repeat an answer.

About the level of control, students mentioned that they got more help than in real life (a protocol model to start with, many resources in the virtual library, feedback on many aspects of their protocol in the e-mail, etc), which make the experience slightly less realistic. In other words, they see the didactical intention behind the feedback they get, event when they come from characters of the game. However, this is not seen as an internal incoherence or a factor of disengagement. A compromise between realism and relevance worked out in this case.

Interactions with characters. We present how students perceived the various interactions on a scale of personification, staring from the most personified character, namely the patients that appear to students in the form of actors on videos.

Patients look real to students "although we know they are actors" or that "they read a text, for some of them". The cues used by student to talk about the authenticity of the patient interactions are numerous: "patients do not say what we ask them to say", "it is not perfect", "not very clear", patients "use their own words", "there are old people telling their life story". According to a student, the "different types of patient are represented (cooperative or not, talkative or not, cranky or not...)". Comparing to real patient interview, one says that "it is the same; we have to sort what they say, and to translate it into medical terms".

As for the e-mail interaction with the experts of the Ethical Committee, some students thinks that "it looks like we talk to our teacher" because it asks for modifications like teachers do, "it looks like they say almost the same thing to everybody in part of the message". It is important to note that students try to compare with a real world reference they do not have. Therefore, realism is judge by what they think is realistic not by their prior experience. Most students say that they cannot judge the credibility of the situation because of that reason. One student thinks that "maybe one gets no explanation when the protocol is rejected, in real life". On the 
other hand, this interaction appears as authentic to most students, for reasons like "it looks real because the mail looks official", "the content is serious".

Considering the interaction with the Hospital, we get a different picture. Most students do not find it authentic, neither realistic nor relevant. From their point of view, "it does not look too serious when all of a sudden everybody gets an answer" (by SMS), " it is not credible to talk to an answering machine", "very bizarre", and it is the "same number for all hospitals". However, one student think that it looks realistic because it is like that when you want to get an appointment with a physician, you often get to talk to an answering machine. Several students think that it would be easier to know what to say with a real person, although more complicated to answer to his/her questions. It seems that it was hard for most students to play the game of this interaction, using an answering machine. On the other hand, only one student mentioned the SMS answer to say that 'to get an answer by SMS is not realistic'.

Finally, we examined how is perceived the interaction with the department of Information of the hospital. Student post a data request on the virtual hospital and get a short e-mail answer with a data file. Some students found it realistic, one because the e-mail is signed. However, most students did not remember to whom they had request their data from, although they were asked about it only one or two hours after they had done it. Only one student reflected about how the system of interaction works. For this student, it was magic when "we got data right away, the file, the patients on videos, and just what we wanted".

\section{Conclusion}

In this work, we probed students' perceptions relative to the authenticity of a learning game environment (see also [2]). The qualitative method used has proven quite successful, as it allowed for the gathering and in-depth analysis of a wide variety of judgments concerning these matters. Overall, our results indicate that students authenticity judgments pertaining to the game LOE can be very complex and specific. In particular, we observed that given cues in the environment could play different, even contradictory, roles in the formation of these judgments. Students could use the same cue to make opposite judgment. This shows that it would be interesting to look for individual traits that could explain some of the authenticity judgments. We also found that, in some instances, unfavourable assessments could be promoted by cues designers had hoped would instead favour authenticity.

We performed a behavioural analysis as follows. The authenticity external (realism) is deduced from the fact that students behave in line with what they would do in "real" life. The internal authenticity was not measured through students actions, but it is analyzed in students' interviews or in discussions between students (not shown here). The didactical authenticity is finally linked to the recognition of the problem or not (wanted by teachers), and the deployment of a strategy to solve it.

We showed how our model (figure 1) can be used to study perceived authenticity. For instance, one may find students who perceive a learning game as externally and internally authentic but not relevant (e.g. students who rely strongly on the teachers and do not see the point of what they do in terms of learning). This model can also be 
used for the design of authentic games. Finally, the model can be used when the authenticity of the game has to be judged by different experts. For instance, external authenticity of the game (sometimes called fidelity) can be assessed by domain experts using more or less well established criteria. Didactical authenticity of the game can be assessed by teachers.

Our proposal is that the design of a game must result in a genuine compromise between the three dimensions of authenticity, namely, realism, coherence and relevance. This authenticity is particularly welcome in a biostatistics course for medical students. Indeed, a mismatch between students' perceptions of their future profession and the content of this type of course justifies an effort to contextualize the learning, i.e. to provide an authentic environment.

Regarding the example of the phone interaction with the hospital, interviews with students confirmed the findings of the analysis of their behaviour: two studies showed a lack of perceived authenticity. Several improvements are planned. To promote the didactical authenticity, we will make the problem more explicit (in particular, require a description of the main objective in the instruction provided by the answering machine). For internal authenticity, we will ask students to give a single call (to avoid repeated calls and responses received by SMS). Finally, for the sake of external authenticity, we will put a professional on the phone from time to time (instead of the answering machine). We hypothesize that these changes will lead to an indication of authenticity observable in student behaviour.

\section{References}

1. Scerbo, M. W., Dawson, S. (2007) High Fidelity, High Performance? Simulation In Healthcare, 2 (4), 224-230.

2. Francis, A. \& Couture, M. (2003) Credibility of a simulation-based virtual laboratory: An exploratory study of learner judgments of verisimilitude. Journal of Interactive Learning Research, 14 (4), 439-464.

3. Shapiro, M. A. Pena-Herborn, J., Hancock, J. T.: Realism, imagination and narrative video games. In: Playing Video Games: Motives, Responses, and Consequences. Eds: Vorderer, P., \& Bryant, J. Mahwah, NJ: Lawrence Erlbaum Associates (2006)

4. Brown, E., Cairns, P.: A grounded investigation of game immersion. Paper presented at the SIGCHI conference on Human factors in computing systems (CHI '04). ACM Press, New York, NY, pp. 1297--1300 (2004)

5. Björk, S., Holopaine, J.: Patterns In Game Design. Charles River Media. pp. 423 (2004)

6. Ney, M., Gonçalves, C., Balacheff, N., Schwartz, C. \& Bosson, J-L. (in press). Phone, Email and Video Interactions with Characters in an Epidemiology Game: towards Authenticity. Lecture Notes on Computer Science (LNCS), Transactions on Edutainment, special issue on Simulation and Gaming in Healthcare.

7. Tseng, S., \& Fogg, B. J. (1999). The Elements of computer credibility. In K. Ehrlich \& W. Newman (Eds.), Proceedings of CHI'99 (Pittsburgh, May 15-20), 80-87. New-York: ACM Press.

8. Chandler, D. (1997). Children's understanding of what is 'real' on television: A review of the literature. [WWW document] Retrieved on April 10, 2010 from http://www.aber.ac.uk/media/Documents/short/realrev.html 
9. Busselle, R. W. \& Greenberg, B. S. (2000). The nature of television realism judgments: a reevaluation of their conceptualization and measurement. Mass Communication and Society, 3, pp. 249-258.

10. Busselle, R., Bilandzic, H.: Fictionality and Perceived Realism in Experiencing Stories: A Model of Narrative Comprehension and Engagement. Communication Theory, 18, 255280 (2008)

11. Gonçalves, C., Ney, M., Balacheff, N., Bosson J-L.: Student's Problem Appropriation in an Epidemiology Game, paper presented at ECGBL 2009, European Conference on GameBased Learning, Graz, Austria (2009)

12. Moher, T. (2006, April) Embedded phenomena: supporting science learning with classroom-sized distributed simulations. Paper presented at ACM CHI 2006 Conference on Human Factors in Computing Systems 2006. pp. 691-700, 2006.

13. Colella V. (2000) Participatory simulations: building collaborative understanding through impressive dynamic modeling, Journal of the Learning Sciences, 9, 471-500.

14. Thomas, S. (2006) Pervasive learning games: Explorations of hybrid educational gamescapes Simulation \& Gaming: An Interdisciplinary Journal, 37, 41-55. 